\title{
À LA RECHERCHE DE LA VOCATION PERDUE. PLAIDOYER POUR L'ENSEIGNEMENT DE LA LITTÉRATURE DES SIÈCLES PASSÉS
}

Cet article ne prétend pas à des ambitions scientifiques : son objectif est de présenter quelques réflexions, basées sur mon expérience personnelle de professeur d'histoire et de littérature française des Lumières et sur mes observations du monde des études et du marché du travail, sur la place actuelle de l'enseignement de la littérature des siècles passés dans le cursus universitaire, et plus précisément en ce que l'on continue d'appeler philologie romane dont il représente toujours parfois au grand dam de la majorité des étudiants — une composante importante, et même centrale. Je tenterai de prouver par la suite que l'enseignement de la littérature des siècles passés possède des aspects pratiques et est essentiel dans le processus de formation des futurs citoyens.

Ceci me semble d'autant plus important que les différentes disciplines rassemblées sous l'enseigne des « humanités » vivent aujourd'hui une crise identitaire face, entre autres, aux nouvelles technologies, qui d'un côté facilitent l'accès au savoir, mais de l'autre, ont fait évoluer les compétences intellectuelles des jeunes, dont les stratégies et habitudes de lecture : généralement, ils ne seraient plus capables de lire de textes de plus de trois pages ni de se concentrer pendant un temps prolongé sur quoi que ce soit ${ }^{1}$. En plus, toute histoire leur semble étrangère

${ }^{1}$ Cf. D. Michalak, I. Koryś, J. Kopeć, Stan czytelnictwa w Polsce w 2015 roku: wstępne wyniki, Biblioteka Narodowa, Warszawa 2016 ; en ligne : <www.bn.org.pl/download/document/1459845698. pdf> [consulté le 30.09.2017]. 
et lointaine, car ils ne vivent que dans le présent, voire dans plusieurs présents « instantanés » et " simultanés ». S'y ajoute encore un facteur qui obsède les sociétés occidentales modernes, à savoir le rejet de tout ce qui semble dépourvu de côté pratique. Telle est aussi l'opinion qui semble se porter à propos de la littérature et de la culture des siècles passés.

Le désintérêt des grands adolescents et des "jeunes adultes $»^{2}$ polonais pour la lecture semble se généraliser. Selon une enquête menée en 2015 par la Bibliothèque nationale polonaise auprès des jeunes de 15 à 19 ans, la moitié seulement aurait lu (ou «feuilleté »!) un livre l'année passée, et seuls 23\% d'entre eux en auraient lu plus de trois ${ }^{3}$. Il faut donc savoir convaincre les étudiants, et ceci même dans les filières de lettres - où ils sont en principe censés s'intéresser à la littérature - , les inciter à la lecture de textes plus longs que quelques pages et ancrés dans de lointaines réalités socio-culturelles.

Enseigner la littérature est d'autant plus difficile - surtout dans les philologies étrangères dont elle devrait pourtant être le fondement — que les étudiants s'en détournent au profit d'autres matières enseignées, à savoir la pratique de la langue et la traduction, les deux étant considérées comme des savoir-faire pratiques, et en tant que tels, de bonnes garanties d'insertion professionnelle.

\section{1. À L'OMBRE DES MATIÈRES PRATIQUES}

Tout enseignant de littérature à l'université doit donc faire face à deux phénomènes préoccupants : $1^{\circ}$ la désaffection des étudiants pour la lecture de textes qu'ils considèrent comme trop longs, $2^{\circ}$ le désintéressement général des étudiants et de l'opinion publique pour tout ce qui, par nature, semble trop théorique ou non pragmatique.

Les résultats d'une petite enquête que j'ai réalisée très récemment le prouvent. J'ai demandé aux étudiants de première année de licence de philologie romane de présenter, de façon anonyme, les motivations qui les avaient amenés à choisir ces études, ainsi que leurs attentes. Les résultats obtenus ne trompent pas : sur seize personnes, trois seulement ont mis en premier lieu le désir d'élargir leurs connaissances et horizons et ont déclaré rechercher dans les études de lettres un développement personnel et intellectuel ; deux autres ont noté, parmi d'autres arguments, qu'elles voulaient connaître " plus ou moins » la littérature française, sans pour autant préciser dans quel but ; les onze autres sont restées muettes concernant tout sujet littéraire ou civilisationnel. Elles n'ont évoqué que l'aspect pratique des

${ }^{2}$ Afin de mieux comprendre la spécificité des « jeunes adultes » $c f$. V. Cicchelli, « Les jeunes adultes comme objet théorique ", Recherches et prévisions 61,2001 , pp. 5-18, <http://www.persee. fr/doc/caf 1149-1590 2001 num 65 1_961> [consulté le 20.10.2017].

${ }^{3}$ D. Michalak, I. Koryś, J. Kopeć, op. cit. 
études philologiques : si elles sont là, c'est parce qu'elles souhaitent apprendre la langue afin d'augmenter leur compétitivité sur le marché du travail. Voilà donc les motivations de la grande majorité des jeunes adultes inscrits en philologie romane à qui $\mathrm{j}$ 'ai affaire ; et les responsables des programmes de cours doivent nolens volens prendre en considération cette demande au moment de la mise en place de l'offre de l'établissement pour les nouvelles admissions.

Certes, on pourrait voir dans les résultats de mon enquête des motivations et des souhaits naïfs et candides de jeunes au seuil de leur parcours universitaire et de leur vie d'adulte. Mais ce n'est pas le cas. Car aux dires des étudiants de troisième année de licence, de ceux qui terminent leur premier cycle de philologie romane, la situation n'est guère différente : « Il y a trop de littératures dans le programme ! ", m'ont-ils déclaré il y a un an, à la fin d'un séminaire de licence qui portait sur le théâtre de la Révolution française. Ce pluriel qu'ils ont utilisé se référait à la répartition traditionnelle de l'enseignement de la littérature en cours magistraux et travaux pratiques, les deux étant complétés par un cours sur la théorie littéraire - d'une durée de deux semestres et uniquement en deuxième année de licence - que l'on propose aux étudiants afin de les doter des outils nécessaires à l'interprétation des textes.

Ces mêmes étudiants se sont plaints de la trop grande " théorisation 》 des travaux pratiques, ainsi que des méthodes "sclérosées» de certains enseignants chargés de leur communiquer des savoirs littéraires. Il n'est pas de mon propos de critiquer les méthodes pédagogiques de mes collègues ni de discuter les attitudes des étudiants qui, me semble-t-il, en restant sous l'influence de l'opinion publique, aspirent tous à faire de belles carrières dans le monde des affaires polonais où la civilisation française des siècles passés ne semble ni utile ni nécessaire 4 .

En tant qu'enseignant de littérature et d'histoire des Lumières, je suis pourtant resté stupéfait face à ces étudiants pour qui la langue ne devrait pas être un but en soi, mais un outil permettant de connaître sensu largo la culture de la France et du monde francophone. Car en fin de compte «l'objet d'études de la philologie est l'ensemble des documents écrits du passé humain, qu'ils soient d'ordre esthétique, religieux, philosophique, historique ou juridique et ces textes sont des monuments témoins d'une culture, d'une civilisation ou d'un individu représentatif de cette culture ou civilisation $»^{5}$. J'éprouvais du mal à avaler la triste vérité : les étudiants, nonobstant tous les efforts des professeurs de littérature et d'histoire,

${ }^{4}$ À Wrocław, la grande majorité des diplômés de philologie romane sont embauchés par les grands centres de téléservices délocalisés depuis quelques années dans la ville, tels Google, HP, UPC, IBM, Nokia ou Crédit Suisse, qui travaillent pour des clients du monde entier et ont donc besoin d'employés francophones. Pour ces entreprises, c'est la compétence langagière qui prime lors de la recherche de nouveau personnel sur le marché local.

${ }^{5}$ C. Rétat-Frydryszak, "La philologie romane en Europe centrale et en France au XIX siècle », Romanica Wratislaviensia LI, Wrocław 2004, p. 76. 
dont les miens, auraient souhaité voir limiter le nombre d'heures consacrées aux « contenus civilisationnels » au profit de l'apprentissage du FLE (français langue étrangère) ou de la traduction. Comme s'ils ne comprenaient pas que traduire un texte, c'est interpréter la culture et l'histoire de son pays, que la traduction n'est pas qu'un exercice lexical et grammatical automatique, et que le traducteur luimême a un grand rôle social et culturel à assumer : celui de faire découvrir et d'expliquer l'Autre et sa culture à ses propres compatriotes. Sinon, l'application Google Translate serait largement suffisante...

\section{2. À LA RECHERCHE DE L'EXPÉRIMENTATION}

Je suis tout à fait d'accord avec l'avis de Mirosław Loba qui, dans un article écrit il y a vingt ans déjà, critiquait les méthodologies post-modernes utilisées alors largement dans l'enseignement philologique, à savoir «l'isolement du texte du contexte historique, l'abandon de l'histoire littéraire au profit de la littérarité, la mise en valeur de la structure linguistique et l'insertion de la psychanalyse ${ }^{6} »$. Dans son analyse de la place de la littérature dans les programmes des philologies à l'aube du XXI ${ }^{\mathrm{e}}$ siècle, il remarque que la littérature ancienne est devenue pour les étudiants un obstacle dans la course au diplôme : « Enseigner le passé à ceux qui ne vivent que dans le rythme des flash du présent, c'est leur rajouter un fardeau supplémentaire $»^{7}$. Lui-même préconise l'interprétation, comme moyen de faire sortir la littérature de l'impasse post-moderne ; et dans un deuxième temps, il propose de la rapprocher de ce que l'on pourrait appeler les cultural studies ${ }^{8}$. Je partage ces opinions.

La littérature, y compris dans les philologies qui, traditionnellement, s'organisent autour d'elle, constitue-t-elle de nos jours « un fardeau » pour les étudiants? Mes observations personnelles, ainsi que celles de mes collègues, semblent aporter une confirmation, même s'il existe des exceptions à la règle.

Or, je suis persuadé que l'enseignement de la littérature, et surtout celle des siècles passés, a toujours un grand rôle social, politique et civilisationnel à jouer non seulement à l'université, mais surtout au niveau national, car il est un des socles du processus d'acquisition par les étudiants-citoyens d'un savoir, et surtout,

${ }^{6}$ M. Loba, «Enseigner la littérature aux étudiants 'postmodernes'», [dans :] Les cahiers de Varsovie : Enseigner la littérature, J. Żurowska (dir.), Wydawnictwa Uniwersytetu Warszawskiego, Warszawa 1998, p. 124.

7 Ibidem.

${ }^{8} \mathrm{Cf}$. M. Loba, «Enseignement de la littérature française dans les facultés de langues étrangères. Nouveaux défis », [dans :] Vers une nouvelle dimension des études romanes, T. Tomaszkiewicz (dir.), Oficyna Wydawnicza LEKSEM, Łask 2006, pp. 35-38. 
d'un $"$ savoir vivre $»{ }^{9}$. Outre les aspects linguistiques et didactiques ${ }^{10}$ de la littérature, sa grande, sa vraie vocation si j'ose dire, est pratique par excellence et consiste à initier les gens inexpérimentés à la vie. La littérature et l'histoire sont toutes les deux des magistri vitae.

Dans un essai datant de 2007, Ottmar Ette affirme « qu'avec la tradition littéraire nous disposons d'un savoir sur la vie qui est précieux pour notre existence présente, qu'il est enfin grand temps de s'investir dans l'analyse de cette dimension des textes, et cela avec une approche de recherche interdisciplinaire qui fait le lien entre les différentes 'sciences de la vie' $\gg{ }^{11}$. Or, selon lui, la littérature en fait partie.

Dans le sillon de la pensée post-moderne qui a refaçonné (ou peut-être défaçonné) il y a quelques décennies l'enseignement de la littérature dans les universités occidentales, on a tenté d'abandonner l'approche historique du texte sous prétexte qu'elle était désuète et archaïque car datant du $\mathrm{XIX}^{\mathrm{e}}$ siècle $^{12}$ :

${ }^{9}$ La littérature moderne quant à elle, d'après de nombreux théoriciens et universitaires, présente une grande différence par rapport à la littérature des siècles passés : avec l'apparition du Nouveau Roman, elle a connu ce que d'aucuns appellent le linguistic turn, qui n'est que la « fin du récit continu de l'événementiel ; [la] fin du personnage comme acteur individualisé de l'histoire ». Il s'agirait désormais de « romans sans personnages, sans intrigue, voire sans auteur ». Dans cette perspective, l'homme n'agirait " plus que dans un monde des signes et des discours ». Cf. W. Asholt, U. Bähler, «Introduction », Revue des sciences humaines 321 (1/2016), titre du volume : « Le savoir historique du roman contemporain », p. 9 ; U. Bähler, « L'historien moderne face au roman historique : positions et postures », Revue des sciences humaines 321 (1/2016), p. 24.

Cette spécificité de la littérature moderne attire l'attention d'un nombre grandissant de chercheurs qui se demandent si, et à quel degré, les ouvrages récents, centrés sur le moi et sa vie intérieure, restant à l'abri de la grande histoire, voire des relations sociales, sont capables de véhiculer de « vrais savoirs ». Cf. Revue des sciences humaines 324 (4/2016), titre du volume : « Les savoirs littéraires ».

10 « [...] le texte littéraire est un moyen de motivation pour l'apprentissage de la langue dans l'enseignement des langues étrangères, le plaisir de la lecture est activement utilisé pour renforcer la motivation : on vise l'identification à des héros, l'empathie avec l'univers fictif, et la prolongation du texte littéraire dans une créativité qui engage l'expression d'une perspective individuelle » (A. Keilhauer, «Impasse ou chemin de traverse ? Le rôle de la littérature dans l'enseignement secondaire et universitaire des langues étrangères, l'exemple du français en Allemagne », [dans :] Enseigner la littérature aujourd'hui, en ligne : <https://oblit.hypotheses.org/category/enseigner-la-litterature-aujourdhui> [consulté le 28.09.2017]).

${ }^{11}$ O. Ette, « Literaturwissenschaft als Lebenswissenschaft. Eine Programmschrift im Jahr der Geisteswissenschaften », [dans :] Enseigner la littérature...

12 J'en ai personnellement fait l'expérience. À l'Institut d'études romanes de l'Université de Wrocław, avant les changements de programmes récents, il y avait en premier cycle un cours obligatoire complétant les TP et les CM de littérature, intitulé « Lectures littéraires ». L'élément central du cours était le travail avec un texte littéraire et son analyse " philologique ». Il semble pourtant que les étudiants n'y étaient pas prêts à ce stade de leur formation. Le cours a vite dérivé du côté du FLE et a abandonné peu à peu toute prétention littéraire, ce que son changement de nom traduit bien : les responsables des programmes de cours l'ont rebaptisé « Lectures » et ont décidé de l'inscrire désormais dans le cursus du français pratique (PNJF). De l'autre côté, mes nombreux séjours 
[...] depuis les années soixante-dix le discours littéraire à l'école et à l'université s'est progressivement modifié en relativisant le biographisme et les études de sources documentaires, pour accorder une plus grande importance aux recherches linguistique, poétique et narratologique qui ont fourni des outils permettant de valoriser autrement les modes du fonctionnement textuel ${ }^{13}$.

À nouveaux temps, nouvelles méthodes, déclarait-on.

Et pourtant, il me semble que c'est justement l'approche socio-culturelle qui rend son âme au texte, lequel n'est au final qu'un témoignage d'une époque, une culture, une société, voire un individu, et reste toujours ancré dans un moment historique bien précis. La littérature serait donc une science expérimentale (sans guillemets !), car elle transmet un " savoir-vivre » par ses nombreux et divers témoignages, ses vécus et ses personnages. Les lecteurs peuvent gagner une expérience de vie sans sortir de chez eux ; ils peuvent regarder dans les livres comme dans un miroir qui reflète d'autres réalités possibles. Il ne reste donc plus qu'à rappeler cette vocation de la littérature en tant que « science de la vie » aux étudiants, mais aussi à une partie des enseignants qui semblent parfois perdus dans les démarches post-modernes ou qui croient que la crise de l'enseignement littéraire s'inscrit dans une crise plus générale, celle des " humanités », sans que personne n'y puisse rien.

Dès lors, pourquoi ne pas changer le cours de littérature en un grand laboratoire expérimental où l'on parlerait du passé, certes, mais aussi du présent et du futur? Car il ne faut pas oublier le caractère cyclique du cours de l'histoire, et par conséquent, de la littérature : les événements et les motifs se répètent, vont et viennent comme la marée, rejetant à chaque fois sur la plage de nouveaux objets, en emportant d'autres, et changeant ainsi le paysage à chaque flux et reflux.

Pour ce faire, il faut oser s'ouvrir à l'interprétation du texte et aux discussions sur des questions parfois difficiles ${ }^{14}$, aux opinions des autres, et toujours s'exprimer dans l'ambiance de respect mutuel et de tolérance qui devraient caractériser

Erasmus en France m'ont appris que les étudiants de lettres françaises n'avaient pas, eux non plus, les compétences historiques nécessaires pour interpréter une œuvre littéraire de Voltaire ou de Montesquieu : à toute question s'éloignant trop de l'aspect purement littéraire, les étudiants - de licence comme de master - soulignaient toujours qu'ils n'étudiaient pas l'histoire ou la sociologie. Fait symptomatique : dans certaines universités où j'ai fait mes stages, il y avait un cours qui s'appelait «Littérature et société », laissant à penser que le social et l'histoire sont largement absents des cours de littérature «normaux ».

${ }^{13} C f$. U. Dethloff, « Statut et fonction de l'explication de texte dans l'enseignement de la littérature française pour germanophones », [dans :] Les cahiers de Varsovie..., p. 47. Dans son article publié dans le même volume, Loba atteste les mêmes tendances en Pologne.

14 À ce propos, je partage l'opinion de Loba : «L'enseignant n'est pas un gardien du sens unique du texte, mais il n'est pas non plus un observateur indolent des interprétations délibérées des étudiants. Son rôle dans le processus de l'appropriation du texte par les élèves peut être très important. [...] Enseigner la littérature, c'est également montrer le parcours dramatique de la décision interprétative dans toute sa complexité et toute l'angoisse devant un texte. La valeur de l'interprétation ne réside pas seulement dans sa puissance régénératrice, mais surtout dans son respect de l'Autre » (M. Loba, « Enseigner la littérature aux étudiants... », p. 128). 
tout homme cultivé et tout débat académique. Il faut vouloir rechercher le contact avec les jeunes, et non seulement exiger de l'effort de leur part, mais aussi s'y astreindre soi-même. Il faut enfin oser transposer le passé et la fiction littéraire dans le présent et dans le vécu des étudiants et des sociétés actuelles, et soumettre ainsi le contenu littéraire à l'épreuve expérimentale. Je suis à ce propos tout à fait d'accord avec Ewa Domańska, qui souligne le rôle pré-figuratif de la littérature ${ }^{15}$ : depuis toujours elle a aidé l'homme à s'imaginer (pré-figurer) d'innombrables mondes alternatifs où il pouvait expérimenter, penser des utopies nouvelles, où il pouvait soumettre à examen toutes ses idées sociales et politiques pour voir si elles étaient réalisables ou quels problèmes elles engendreraient. Dès le début, la littérature a véhiculé toutes les aspirations de l'humanité à travers les époques et les cultures : tel est son vrai pouvoir.

Dans cette perspective, je lance un appel aux académiciens littéraires : Experiri aude!

\section{PRISONNIÈRE DES IDÉES REÇUES ?}

L'enseignement de la littérature, dont le but est de présenter le fonctionnement de l'individu, voire d'un groupe d'individus, dans la société à un moment historique donné, semble être - a contrario de toutes les idées reçues que l'on entend prononcer dans les médias et qui sont après répétées mécaniquement par une partie des étudiants - un très bon cadre pour développer chez les jeunes au seuil de leur vie d'adulte des compétences sociales et professionnelles dites molles, que les employeurs recherchent chez les nouveaux salariés. Selon une enquête menée pour le Forum Économique Mondial, ces compétences seront en 2020 : la résolution de problèmes complexes, l'esprit critique, la créativité, le management, l'esprit d'équipe, l'intelligence émotionnelle, le jugement et la prise de décision, le sens du service, la négociation, la flexibilité ${ }^{16}$. Il semble que seuls le management et peut-être le sens du service soient difficiles à atteindre en classe ; par contre, toutes les autres compétences restent envisageables et praticables en cours de littérature et découlent directement de la vocation critique, relativiste et créative de ce domaine. Qui plus est, selon les statistiques citées dans un article publié dans la version électronique du journal Gazeta Prawna, 70\% des employeurs licencient leurs salariés quand ceuxci n'ont pas assez de compétences molles, parce qu'il devient difficile de travailler

${ }^{15} C f$. E. Domańska, « Sprawiedliwość epistemiczna w humanistyce zaangażowanej », Teksty Drugie 1, 2017, pp. 41-59.

${ }^{16}$ Cf. <https://www.weforum.org/agenda/2016/01/the-10-skills-you-need-to-thrive-in-thefourth-industrial-revolution/> [consulté le 29.09.2017]. 
avec eux. Aux yeux de ces mêmes employeurs, le manque de compétences dures et de savoir-faire chez un employé n'est que secondaire ${ }^{17}$.

Contrairement donc à ce que l'on répète dans les médias et même récemment au Ministère de la Science et de 1'Enseignement supérieur polonais ${ }^{18}$, les entreprises de notre époque changeante et mondialisée préfèrent embaucher des diplômés qui possèdent des compétences transversales, par opposition aux « savoirs durs » spécifiques à une discipline scientifique ou professionnelle définie. Car, ceux-ci sont faciles à enseigner "sur place », au sein des entreprises, tandis que les compétences transversales ne s'acquièrent ni vite ni facilement. Or, il s'agit de compétences de différents ordres : $1^{\circ}$ intellectuel (exploiter l'information, localiser et résoudre un problème, exercer son jugement critique, penser créatif), $2^{\circ}$ méthodologique (se donner des méthodes de travail efficaces, exploiter les technologies de l'information et de la communication), $3^{\circ}$ personnel et social (actualiser son potentiel, coopérer en équipe) et enfin $4^{\circ}$ communicatif (communiquer de façon efficace et appropriée) ${ }^{19}$. Ce sont exactement les compétences que les philologies offrent à leurs étudiants, mais qui, aux yeux de l'opinion publique, restent largement dévalorisées, car on les croit acquises à la sortie du lycée, ce qui est dans la plupart des cas naturellement faux. Et c'est d'ailleurs cette fausse idée qui aiguille les nouveaux étudiants vers des savoir-faire et des compétences dures.

\section{DU CÔTÉ DE CHEZ L’AUTRE}

Je vais finir mes considérations sur le rôle que l'enseignement de la littérature des siècles passés a toujours à jouer dans les filières philologiques par une constatation tout à fait évidente mais à laquelle on pense rarement : le temps passé par l'étudiant à l'université est très souvent l'unique époque de sa vie où il est invité à développer son sens critique, à penser vraiment librement ; et dans la plupart des cas, il n'en a pas l'habitude. En effet, rares sont les écoles secondaires qui laissent de la place aux libres discussions sur les textes et leur sens (principalement pendant les cours de polonais, qui semblent les seuls à se prêter à l'interprétation). Les

${ }^{17}$ Cf. $<$ http://serwisy.gazetaprawna.pl/praca-i-kariera/artykuly/1071598,humanisci-na-rynku-pracy-umiejetnosci-miekkie.html> [consulté le 29.09.2017].

${ }^{18}$ Cf. <http://serwisy.gazetaprawna.pl/edukacja/artykuly/1071937,reforma-gowina-likwidacja-kierunkow-studiow.html $>$ [consultéle30.01.2018]; $<$ http://wyborcza.pl/7,75398,21457641, wicepremier-gowin-dla-wyborczej-chce-podniesc-poprzeczke.html> [consulté le 30.01.2018].

19 Je m'appuie sur la classification présentée par Denis Berthiaume de l'Université de Lausanne lors de sa communication au colloque « Promosciences », à l'Université Nantes les 21-23 mars 2012 ; la communication est consultable en ligne sous le lien : $<$ https://www.google.pl/url?sa $=t \& r c t=j \& q=\& e s-$ $\mathrm{rc}=\mathrm{s} \&$ source $=$ web $\& \mathrm{~cd}=6 \& \mathrm{cad}=\mathrm{rja} \&$ uact $=8 \& \mathrm{ved}=0 \mathrm{ahUKEw}$ ifiY Cc $2 \mathrm{sfVAhUGZ} 1 \mathrm{AKHU}-$ HJDgsQFghMMAU\&url=http\%3A\%2F\%2Fspiralconnect.univ-lyon1.fr\%2Fspiral-files\%2Fdownload\%3Fmode\%3Dinline\%26data\%3D3399915\&usg=AFQjCNE2-1rcNSTHCZ-yNwTMj9BivFwZkQ $>$ [consulté le 8.08.2017]. 
raisons sont multiples, mais les plus importantes restent, semble-t-il, le manque de temps pour réaliser les contenus des programmes d'enseignement imposés par le Ministère de l'Éducation nationale, et le souci des enseignants de transmettre aux collégiens et aux lycéens les informations (et interprétations !) conformes aux réponses attendues par les correcteurs lors des examens finaux.

La famille non plus, et pour maintes raisons, ne sert pas de forum démocratique d'échange des idées. Aussi les jeunes se construisent-ils leur identité « en ligne », sur des réseaux sociaux, et très souvent " à l'aveuglette », car il leur est difficile de discerner le vrai du faux dans la marée d'informations qui débordent de l'écran. Nous vivons tous dans l'ère post-véritée $e^{20}$ les gens inexpérimentés peuvent s'y perdre facilement. Il faut les aider à s'en sortir. Et où le faire, sinon à l'université, en cours de littérature ou d'histoire, où tout devrait se prêter à l'interprétation, où tout devrait « se discuter »? Cette approche critique devrait tendre vers un objectif : la vérité des choses.

Même s'il peut sembler qu'initier les étudiants à penser par eux-mêmes est un peu tardif à ce stade de leur développement intellectuel et émotionnel, il faut relever ce défi. En tant qu'universitaires, nous y sommes obligés, c'est une dette scientifique, mais aussi civique et éthique, que nous devons acquitter auprès de la société. Former de futurs citoyens, conscients de leurs droits, mais aussi conscients des droits de l'Autre, sachant lui parler et résoudre les problèmes de concert avec lui : voilà notre engagement, nous ne pouvons pas le rejeter.

L'université reste pour la plupart des étudiants, qui sont sur le point de s'émanciper socialement et politiquement, le seul endroit - et ce caractère unique est à souligner - où ils sont invités à développer chez eux un relativisme cognitif, à se confronter, dans un esprit démocratique et égalitaire, aux opinions et aux vécus de l'Autre ; et cet Autre peut être à la fois leur professeur, leur camarade ou un personnage fictif issu d'un roman. Un débat organisé en classe ou des travaux écrits individuellement sur un sujet imposé par l'enseignant ne sont pas que des exercices purement érudits, créatifs ou langagiers : leur premier rôle dans le cadre universitaire devrait être à mes yeux d'inviter les jeunes à se construire une conception du monde et une identité émotionnelle personnelles, à développer chez eux l'empathie dont ils se serviront dans leur vie d'adulte. Bref, l'université devrait être pour eux l'endroit d'émulation intellectuelle dont ils ont tous besoin à leur âge. Car par la suite, il n'y en aura très probablement plus d'autres.

Dans le monde mondialisé et « instantané », imprégné de culture matérialiste, dans ce monde post-moderne qui est le nôtre, où beaucoup de gens ne sont plus capables de vivre leur vie " normalement ", se cachant derrière l'écran de leur

${ }^{20} C f$. W. Audureau, «Faits alternatifs, fake news, post-vérité... petit lexique de la crise de l'information », Le Monde, 05/01/2017, en ligne : <http:/www.lemonde.fr/les-decodeurs/article/2017/01/25/ faits-alternatifs-fake-news-post-verite-petit-lexique-de-la-crise-de-1-information_5068848_4355770. html\#ancre post-v\%C3\%A9rit $\% \mathrm{C} 3 \% \mathrm{~A} 9>$; pour la définition de l'《ère post-vérité », cf. <https:// fr.wikipedia.org/wiki/\%C3\%88re post-v\%C3\%A9rit\%C3\%A9> [consulté le 30.09.2017]. 
ordinateur et se créant sans cesse de nouvelles identités, enseigner les compétences sociales telles que l'empathie, le dialogue avec l'Autre, l'esprit critique ou l'esprit d'équipe s'avère un grand défi social et politique à relever. Et quand on y pense bien, malgré les nombreuses réserves que l'on peut formuler à son encontre, l'enseignement de la littérature (et de la civilisation) des siècles passés peut s'y prêter facilement.

Dans cette perspective, la démocratisation de l'enseignement supérieur et la popularité des études philologiques est, du point de vue des intérêts de la société, une chance et non pas un obstacle à l'insertion des jeunes dans le monde du travail, à condition que l'on en use avec prudence et intelligence. Il revient aux enseignants universitaires de (re)prendre l'initiative. D'autant plus qu'ils sont les seuls responsables du contenu et de la réalisation de leurs programmes de cours, contrairement aux enseignants des niveaux précédents qui sont contraints de respecter les programmes imposés par le ministère.

\section{VOCATION RETROUVÉE}

Le grand objectif de l'enseignement de la littérature des siècles passés serait à mes yeux l'autonomisation des jeunes et leur émancipation intellectuelle, afin de former de futurs citoyens respectueux et responsables les uns des autres.

En tant que spécialiste de l'époque des Lumières, je sens peut-être cette responsabilité plus fortement que mes collègues. D'autant plus que nous vivons en Occident une sorte de crise générale des valeurs. Le cours des choses a pris récemment dans beaucoup de pays un tournant nationaliste et xénophobe ; les valeurs jusque-là indiscutables, telles la démocratie libérale, la liberté de parole ou la tolérance, sont mises en doute par les politiciens et par l'opinion des masses que l'on confond souvent avec l'opinion publique. Les valeurs fondamentales de la culture européenne que les philosophes des Lumières nous ont léguées semblent aujourd'hui menacées. En outre, la culture matérialiste et cyberspatiale dans laquelle nous vivons, ainsi que la poursuite de l'argent et du divertissement ont aveuglé beaucoup de gens, surtout les jeunes, les détournant de ce qui est vraiment important : le bonheur commun. La condition de tout bonheur étant la vérité, il faut apprendre aux jeunes comment et où la retrouver. Il faut leur montrer comment être philosophes dans leur vie quotidienne : leur enseigner à prendre « pour vrai ce qui est vrai, pour faux ce qui est faux, pour douteux ce qui est douteux, et pour vraisemblable ce qui n'est que vraisemblable $»^{21}$. Sinon, perdus dans la réalité des post-vérités et incapables de discerner le vrai du faux, ils ne sauront

${ }^{21}$ C.Ch. Dumarsais, «Philosophe », [dans :] Encyclopédie, ou dictionnaire raisonné des sciences des arts et des métiers, D. Diderot, J. d'Alembert (dir.), <http://portail.atilf.fr/cgi-bin/getobject_?a.92:109./var/artfla/encyclopedie/textdata/IMAGE/> [consulté le 15.10.2017]. 
jamais sortir de leur « minorité » intellectuelle qui, comme le disait Emmanuel Kant, consiste « dans l'incapacité de se servir de [leur] intelligence sans être dirigé par autrui $»^{22}$.

Former un être qui, dans ses actions, se base sur son entendement personnel et autonome, qui est conscient de son engagement social et qui sait prendre la responsabilité du bonheur commun : voici une grande vocation de l'université réalisable en cours de littérature. La cause en vaut le risque ; nous n'avons qu'à oser.

\section{IN SEARCH OF LOST VOCATION. DEFENSE OF THE LITERATURE OF PAST TIMES AT THE ROMANCE PHILOLOGY}

\section{Summary}

The aim of this article is to discuss the place of the literature of past centuries in the romance philology teaching programmes. I accentuate the social importance of literary subjects at the university in the making of future citizens responsible for the common good, as well as the social importance of literature itself. Furthermore, I emphasize the role of scholars who are assumed not only to bring knowledge to their students, but also to teach them essential social skills. The analysis is based on my ten-year academic experience of specialist in the history of literature and history of the French Enlightenment.

Key words: university, academia, vocation, literature, teaching, emancipation, philology.

22 E. Kant, Qu'est-ce que les Lumières ?, texte intégral de la traduction française disponible sous le lien : <https://fr.wikisource.org/wiki/Qu\%E2\%80\%99est-ce_que_les_Lumi\%C3\%A8res_\%3F> [consulté le 15.10.2017]. 